\title{
Evaluation of Ac-Lys ${ }^{0}$ (IRDye800CW)Tyr ${ }^{3}$-octreotate as a novel tracer for SSTR $_{2}$-targeted molecular fluorescence guided surgery in meningioma
}

\author{
Bianca M. Dijkstra ${ }^{1}$ (D) - Marion de Jong ${ }^{2}$ - Marcus C. M. Stroet ${ }^{2,3}$ - Fritz Andreae ${ }^{4}$. Sebastiaan E. Dulfer ${ }^{1}$. \\ Marieke Everts $^{5}$. Schelto Kruijff ${ }^{6}$ Julie Nonnekens ${ }^{2,3}$ - Wilfred F. A. den Dunnen ${ }^{7}$. Frank A. E. Kruyt ${ }^{5}$ (D) \\ Rob J. M. Groen ${ }^{1}$ (D)
}

Received: 26 January 2021 / Accepted: 12 March 2021 / Published online: 26 March 2021

(C) The Author(s) 2021, corrected publication 2021

\begin{abstract}
Purpose Meningioma recurrence rates can be reduced by optimizing surgical resection with the use of intraoperative molecular fluorescence guided surgery (MFGS). We evaluated the potential of the fluorescent tracer 800CW-TATE for MFGS using in vitro and in vivo models. It targets somatostatin receptor subtype $2\left(\mathrm{SSTR}_{2}\right)$, which is overexpressed in all meningiomas. Methods Binding affinity of $800 \mathrm{CW}$-TATE was evaluated using $\left[{ }^{177} \mathrm{Lu}\right] \mathrm{Lu}$-DOTA-Tyr ${ }^{3}$-octreotate displacement assays.

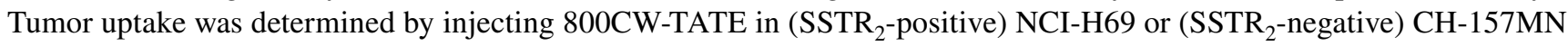
xenograft bearing mice and FMT2500 imaging. SSTR $_{2}$-specific binding was measured by comparing tumor uptake in NCI-H69 and CH-157MN xenografts, blocking experiments and non-targeted IRDye800CW-carboxylate binding. Tracer distribution was analyzed ex vivo, and the tumor-to-background ratio (TBR) was calculated. SSTR 2 expression was determined by immunohistochemistry (IHC). Lastly, 800CW-TATE was incubated on frozen and fresh meningioma specimens and analyzed by microscopy.

Results 800CW-TATE binding affinity assays showed an $\mathrm{IC}_{50}$ value of $72 \mathrm{nM}$. NCI-H69 xenografted mice showed a TBR of 21.1. 800CW-TATE detection was reduced after co-administration of non-fluorescent DOTA-Tyr ${ }^{3}$-octreotate or administration of IRDye $800 \mathrm{CW}$. CH-157MN had no tumor specific tracer staining due to absence of $\mathrm{SSTR}_{2}$ expression, thereby serving as a negative control. The tracer bound specifically to $\mathrm{SSTR}_{2}$-positive meningioma tissues representing all WHO grades.

Conclusion $800 \mathrm{CW}$-TATE demonstrated sufficient binding affinity, specific SSTR $_{2}$-mediated tumor uptake, a favorable biodistribution, and high TBR. These features make this tracer very promising for use in MFGS and could potentially aid in safer and a more complete meningioma resection, especially in high-grade meningiomas or those at complex anatomical localizations.
\end{abstract}

Keywords $800 \mathrm{CW}$-TATE $\cdot$ Intra-operative guidance $\cdot$ Meningioma $\cdot$ Molecular fluorescence guided surgery $\cdot$ Somatostatin receptor subtype 2

Abbreviations
$\begin{array}{ll}\text { 800CW-TATE } & \text { Ac-Lys }^{0} \text { (IRDye800CW) } \text { Tyr }^{3} \text {-octreotate } \\ \text { BBB } & \text { Blood-brain-barrier } \\ \text { DAB } & \text { 3,3-Diaminobenzidine } \\ \text { DAPI } & \text { 4',6-Diamidino-2-phenylindole }\end{array}$

Frank A. E. Kruyt and Rob J. M. Groen authors share last authorship.

Rob J. M. Groen

r.j.m.groen@umcg.nl

Extended author information available on the last page of the article
DOTA

DOTA-TATE
DOTA-TOC
FCS
FFPE
GE MRI

$\mathrm{H} \& \mathrm{E}$

HRP

IHC

MFGS

MFI
1,4,7,10-Tetraazacyclododecane-1,4,7,10tetraacetic acid DOTA-Tyr ${ }^{3}$-octreotate DOTA-Phe ${ }^{1}$-Tyr ${ }^{3}$-octreotide Fetal calf serum Formalin fixed paraffin embedded Gadolinium enhanced magnetic resonance imaging Hematoxylin \& eosin Horse radish peroxidase Immunohistochemistry Molecular fluorescence guided surgery Mean median fluorescence intensity 


$\begin{array}{ll}\text { PBS } & \text { Phosphate buffered saline } \\ \text { SEM } & \text { Standard error of mean } \\ \text { SSTR }_{2} & \text { Somatostatin receptor subtype 2 } \\ \text { TBR } & \text { Tumor-to-background ratio } \\ \text { WHO } & \text { World health organization }\end{array}$

\section{Introduction}

Meningiomas comprise one third of all intracranial tumors in adults. Although these tumors are most often benign, the compression of intracranial neural structures by a growing meningioma can result in serious neurological signs and symptoms, urging for surgical treatment. The most recent WHO criteria distinguish three grades of meningiomas based on histopathological characteristics: WHO grade I, II and III [1]. Surgical treatment is only curative by complete tumor resection. Unfortunately, anatomical localization and local invasion can pose surgical challenges, leading to high recurrence rates [2-5]. Even in benign (WHO grade I) meningiomas, 10-year progression free survival decreases significantly when comparing incomplete resection to gross total resection: $37 \%$ vs $61 \%$, respectively [2]. Molecular fluorescence guided surgery (MFGS) by means of intraoperative guidance could facilitate optimal resection while at the same time aiming to preserve neurological function.

MFGS applies a tumor-targeting tracer that is usually administered intravenously prior to surgery. The tracer consists of two parts: a component specifically binding to a selected tumor-specific biomarker, and a fluorescent dye. The biomarker should be overexpressed on tumor tissue to obtain a sufficiently high tumor-to-background ratio (TBR), which is essential for optimal visualization and thus optimal resection. The fluorescent signal emitted by the tracer allows for intraoperative detection with a neurosurgical microscopic system. IRDye800CW is often applied in tracer development. This dye fluoresces in the near-infrared spectrum, which results in less interference of tissue auto-fluorescence and shows a superior tissue penetration depth. Consequently, such a molecular targeted meningioma-specific tracer may have higher sensitivity, specificity and superior penetration depth compared with currently available dyes such as 5-ALA, ICG or fluorescein.

In the present study, we developed a possible tracer for meningiomas that is directed against the somatostatin receptor subtype $2\left(\mathrm{SSTR}_{2}\right)$. We and others previously demonstrated overexpression of $\mathrm{SSTR}_{2}$ on all meningioma grades, making it a promising biomarker for binding [6-11]. Tyr ${ }^{3}$-octreotate, a previously generated peptideanalogue with a high affinity for SSTR 2 [12], served as the meningioma specific binding part. Subsequent labelling with IRDye800CW yielded Ac-Lys ${ }^{0}$ (IRDye800CW) $\mathrm{Tyr}^{3}$ octreotate (800CW-TATE). Here, we investigated the use of
800CW-TATE for suitability as a tracer in MFGS for meningiomas. Since both octreotate and IRDye $800 \mathrm{CW}$-tracers have been already applied in patients [13-16], this tracer is a promising candidate for clinical translation in meningioma surgery.

\section{Materials and methods}

A full description of the materials and methods is available as online resource.

\section{Cell culture}

Small cell lung cancer NCI-H69 (ECACC) and meningioma CH-157MN cells (dr. Gillespie, University of Alabama) were maintained as described previously [17-20].

\section{Immunohistochemistry on formalin fixed paraffin embedded (FFPE) sections}

SSTR $_{2}$ expression in $\mathrm{H} 69$ and $\mathrm{CH}-157 \mathrm{MN}$ cells in vitro was determined by analyzing sections of Cellient blocks stained for $\mathrm{SSTR}_{2}$ (MAB4224, R\&D systems).

Separate consecutive FFPE sections of tissue obtained from animal studies were used for staining with either 4',6-diamidino-2-phenylindole (DAPI), hematoxylin and eosin (H\&E), or SSTR 2 expression analysis (AC-0162RUO, SanBio) [7].

\section{Xenograft mice models}

H69 (5 million cells) or $\mathrm{CH}-157 \mathrm{MN}$ cells (1.5 million cells) were inoculated in BALB/c-nu mice (Janvier Laboratories) by subcutaneously injecting between the scapulae [17-19].

\section{Displacement assays}

Snap frozen slices of resected H69 xenografts known to abundantly express SSTR 2 [17-19] were incubated with $10^{-9} \mathrm{M}\left[{ }^{177} \mathrm{Lu}\right] \mathrm{Lu}$-DOTA-Tyr ${ }^{3}$-octreotate $\left(\left[{ }^{177} \mathrm{Lu}\right] \mathrm{Lu}\right.$-DOTATATE; IDB Holland) together with a concentration range of $10^{-6}$ to $10^{-12} \mathrm{M}$ 800CW-TATE (piCHEM; Online Resource 1), or DOTA-TATE (Bachem). Sections were subsequently washed, and activity was detected with the Cyclone (PerkinElmer). Data was analyzed as described previously [21].

\section{Tracer uptake, biodistribution and imaging}

Mice bearing xenografts $\left(500 \mathrm{~mm}^{3}\right)$ were given an intravenous bolus with indicated doses of compounds by retroorbital injection into the venous sinus under general anesthesia. Tumor uptake of 800CW-TATE was determined by 
longitudinal imaging for 1,2 , and $4 \mathrm{~h}$ post tracer injection using the FMT2500 (PerkinElmer). H69 xenografted mice were injected with either $800 \mathrm{CW}-\mathrm{TATE}(3 \mu \mathrm{g}, 1.36 \mathrm{nmol})$, DOTA-TATE $(3 \mathrm{mg}, 2.2 \mu \mathrm{mol})$ and $800 \mathrm{CW}$-TATE $(3 \mu \mathrm{g}, 1.36 \mathrm{nmol})$, or IRDye $800 \mathrm{CW}$ carboxylate $(1.5 \mu \mathrm{g}$, $1.36 \mathrm{nmol}$; LI-COR Biosciences). CH-157MN xenograft bearing mice were injected with 800CW-TATE ( $3 \mu \mathrm{g}$, $1.36 \mathrm{nmol})$. Mice were terminated for further postmortem evaluation four hours post injection. Xenografts, organs and fluids were harvested and macroscopically imaged using the PEARL scanner (LI-COR Biosciences) to determine ex vivo tumor uptake and biodistribution. Tissues were sectioned in sequential slides, and tracer fluorescence was imaged using an Odyssey CLx (LI-COR Biosciences). Consecutive slides were processed for fluorescence microscopy, anti-SSTR 2 (AC-162RUO), and H\&E staining as described above.

Statistical significance was tested using a one-way ANOVA with Bonferroni post-hoc analysis. Fluorescence specificity values are reported as mean \pm SEM. The TBR was defined as the ratio of the mean fluorescence intensity (MFI) of the tumor, and the MFI of brain tissue. TBRs are shown as mean with $95 \%$ confidence intervals in brackets.

\section{Post-mortem intra-operative molecular fluorescence guided surgery}

One H69 xenograft bearing mouse was intravenously injected with 800CW-TATE for post-mortem fluorescence guided surgery. The xenograft was first resected using white light guidance, followed by fluorescence guided resection of residual tissue with the SurgVision (SurgVision BV, 't Harde). The TBR was calculated by dividing the MFI of the tumor by the MFI of surrounding muscle tissue.

\section{Analyses of human meningioma specimens}

Ten meningioma specimens resected between 2006 and 2012 were available, and most of these have been described previously: two WHO grade III meningiomas have been added to the current dataset [7]. Frozen sections were incubated with $10^{-6} \mathrm{M} 800 \mathrm{CW}-\mathrm{TATE}$ for one hour, mounted with Prolong Antifade, and imaged using an inverted microscope. Sequential sections were stained with either H\&E or anti-SSTR 2 (MAB4224). For whole specimen analysis, two freshly resected meningioma specimen were cut into $3 \mathrm{~mm}$ sections and incubated for four hours using DMEM/ F12, supplemented with $1 \%$ penicillin/streptomycin and $5 \times 10^{-8}$ M $800 \mathrm{CW}-\mathrm{TATE}$. Sections were cut for a DAPI, $\mathrm{H} \& \mathrm{E}$ and anti-SSTR 2 (AC-0162RUO) stain as described above.

\section{Results}

\section{Binding affinity of $800 \mathrm{CW}$-TATE}

To determine binding affinity of 800CW-TATE, different tracer concentrations were applied in displacement assays with radiolabeled $\left[{ }^{177} \mathrm{Lu}\right] \mathrm{Lu}$-DOTA-TATE on H69 xenograft frozen sections. DOTA-TATE has a high binding affinity for $\mathrm{SSTR}_{2}$ as was shown in previous work [22]. 800CW-TATE had an $\mathrm{IC}_{50}$ of $72 \mathrm{nM}$ and unlabeled DOTA-TATE, as a control, had an $\mathrm{IC}_{50}$ of $2.5 \mathrm{nM}$. The $\mathrm{IC}_{50}$ of $800 \mathrm{CW}-\mathrm{TATE}$ was considered sufficient to continue tracer evaluation. (Online Resource 2).

\section{Tumor uptake and biodistribution of $800 \mathrm{CW}$-TATE in mice}

To determine in vivo tumor uptake and tissue distribution of 800CW-TATE we used $\mathrm{H} 69$ and $\mathrm{CH}-157 \mathrm{MN}$ xenograft mouse models. Tracer fluorescence was clearly detected in the H69 tumor and kidneys within one hour after injection of 800CW-TATE. After four hours, a more homogeneous uptake was observed, evidenced by lower levels of fluorescence in intratumoral hotspots (Fig. 1a, top panel).

Quantification of the fluorescent tracer signal showed stabilized tumor uptake over time, since the signal did not further increase over time (Fig. 1b). SSTR 2 selective tumor uptake was confirmed by saturating (blocking) $\mathrm{SSTR}_{2}$ using DOTA-TATE, followed by 800CW-TATE administration. The tracer signal appeared reduced at all-time points in vivo, although to a small extent (Fig. 1a, second panel), which is likely caused by non-specific uptake in the mouse skin, as also described further on. Additionally, IRDye800CW (without the SSTR $_{2}$-targeting TATE) did not show tumor-specific fluorescence. (Fig. 1a, third panel).

Surprisingly, 800CW-TATE showed much less tumor fluorescence in $\mathrm{CH}-157 \mathrm{MN}$ xenografts compared with H69 xenografts. (Fig. 1a, bottom panel) This is explained by the absence of $\mathrm{SSTR}_{2}$ expression in CH-157MN xenografts, which was unexpected since in vitro cultured cells did have $\mathrm{SSTR}_{2}$ expression. (Online Resource 3) The CH-157MN model thus provided an additional negative control for $\mathrm{SSTR}_{2}$-mediated tumor uptake. Moreover, it also demonstrated that the $800 \mathrm{CW}$-TATE signal likely originated from the skin covering the tumor, thereby distorting the in vivo tumor signal.

The biodistribution of 800CW-TATE was determined ex vivo in resected xenograft and mice tissues. Fluorescent imaging showed that 800CW-TATE mainly accumulated in H69 xenografts, kidneys, urine, liver, and to a lesser extent in the skin (Fig. 2a, b). Fluorescence was very low or absent in the brain, skull, muscle and blood (Fig. 2a, b; 
Fig. 1 In vivo optical imaging of 800CW-TATE in H69 and $\mathrm{CH}-157 \mathrm{MN}$ xenografted mice. a Representative images of $\mathrm{H} 69$ xenograft bearing mice injected with 800CW-TATE showed accumulation of fluorescence in the tumor after one and two hours, and stabilized four hours post injection (top panel). Imaging of $\mathrm{SSTR}_{2}$-positive H69 xenograft bearing mice injected with DOTA-TATE and 800CW-TATE (second panel) or IRDye800CW (third panel), and $\mathrm{SSTR}_{2}$-negative $\mathrm{CH}$ -

$157 \mathrm{MN}$ xenograft bearing mice injected with $800 \mathrm{CW}-\mathrm{TATE}$ (bottom panel) showed reduced accumulation of fluorescence compared with mice injected with 800CW-TATE. b The coadministration of DOTA-TATE as a block slightly reduced 800CW-TATE fluorescence at all timepoints, possibly due to uptake of 800CW-TATE in the skin, thereby distorting the signal. $\mathrm{CH}-157 \mathrm{MN}$ xenografts did not show tracer uptake due to the absence of $\mathrm{SSTR}_{2}$ expression. IRDye800CW showed a significantly reduced tumor fluorescence in H69 xenografts
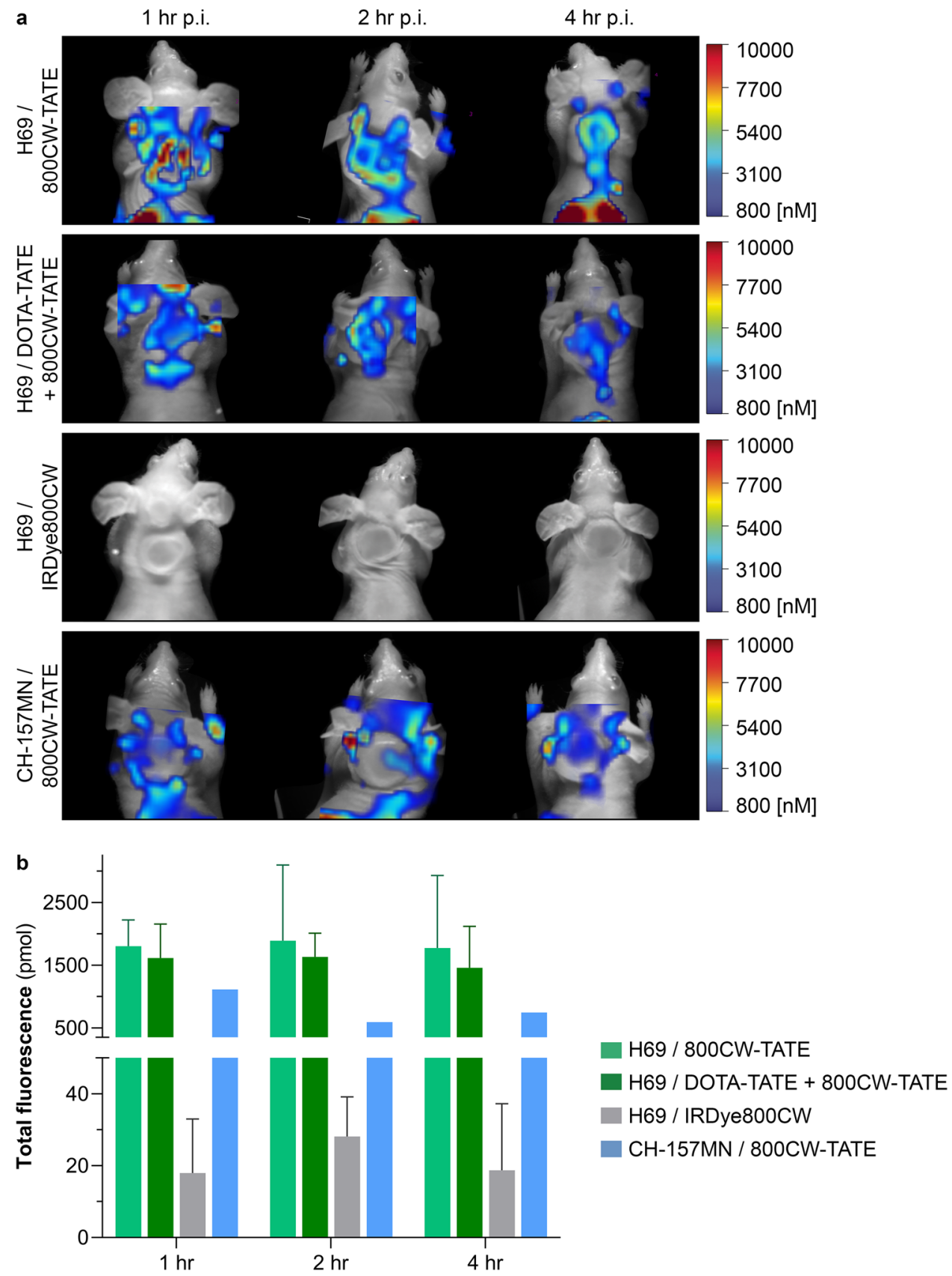

Online Resource 4). This was also reflected by calculating the TBR, which was 21.1 (16.4-26.7). The TBR of other relevant structures considering the anatomical localization of meningiomas was high as well. (Fig. 2c).

Tracer uptake specificity could be determined by comparing tumor fluorescence intensity between treatment groups. The DOTA-TATE block resulted in a $57.8 \pm 5.2 \%$ reduction in tracer uptake. Furthermore, compared with 800CW-TATE-injected H69 xenograft bearing mice, IRDye800CW carboxylate-injected $\mathrm{H} 69$ xenografted mice had $83.4 \pm 4.8 \%$ reduction, and $\mathrm{SSTR}_{2}$ negative $\mathrm{CH}-157 \mathrm{MN}$ xenograft bearing mice showed a reduction of $78.4 \pm 4.8 \%$ tumor uptake. (Fig. 2d).

At the macroscopic level a homogenous tracer distribution in $\mathrm{H} 69$ xenografts was demonstrated after tumor resection and subsequent imaging (Fig. 3a). In the same xenograft, fluorescence microscopic analyses showed presence of 800CW-TATE mostly at the cell membrane and cytoplasm. In addition, membranous $\mathrm{SSTR}_{2}$ expression was confirmed by immunohistochemistry (IHC; Fig. 3b). Although $\mathrm{CH}-$ $157 \mathrm{MN}$ cells showed $\mathrm{SSTR}_{2}$ expression in vitro (Online Resource 3), CH-157MN xenografts showed no $\mathrm{SSTR}_{2}$ 
Fig. 2 Ex vivo optical imaging of resected tissues from $\mathrm{H} 69$ or $\mathrm{CH}-157 \mathrm{MN}$ xenograft bearing mice. a $H 69$ xenografted mice treated with $800 \mathrm{CW}$-TATE were examined for tracer tissue distribution four hours postinjection. Fluorescence uptake was measured with the PEARL and demonstrated uptake mainly in the tumor, skin, kidneys, urine, and liver. In contrast, the fluorescence intensity was (very) low in the brain, skull, muscle, and blood. b The indicated xenografted mice were injected with $800 \mathrm{CW}$ TATE in absence or presence of DOTA-TATE or with non-targeted IRDye800CW. Quantification of fluorescence intensity revealed the indicated biodistribution. c Tissue-tobrain ratios for 800CW-TATE were calculated for the indicated tissues, with a TBR of 21.1. d Tumor fluorescence significantly decreased in the groups co-administrated with DOTATATE, the groups administered with IRDye800CW carboxylate, and in $\mathrm{CH}-157 \mathrm{MN}$ xenografts ( $\mathrm{p}<0.0001$ ). ns, not significant; $\Delta$, difference

a

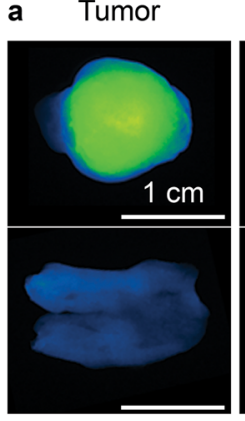

Brain

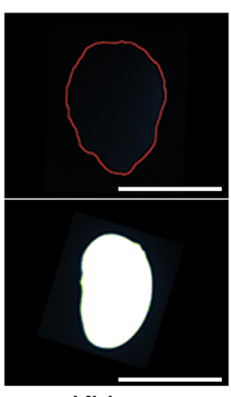

Kidney
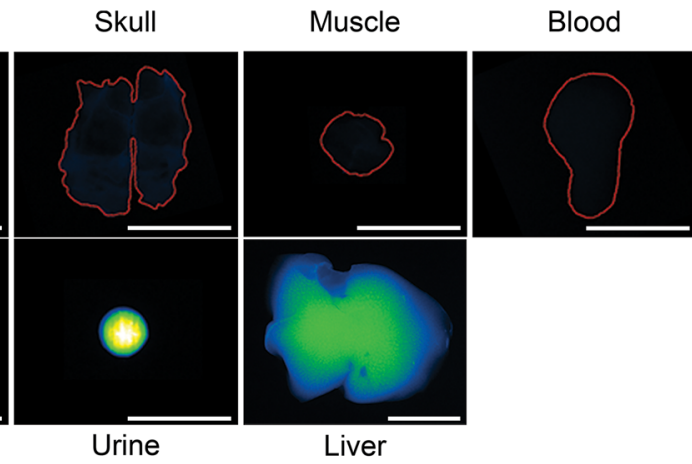

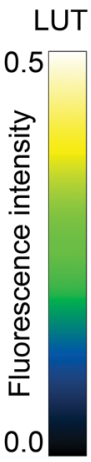

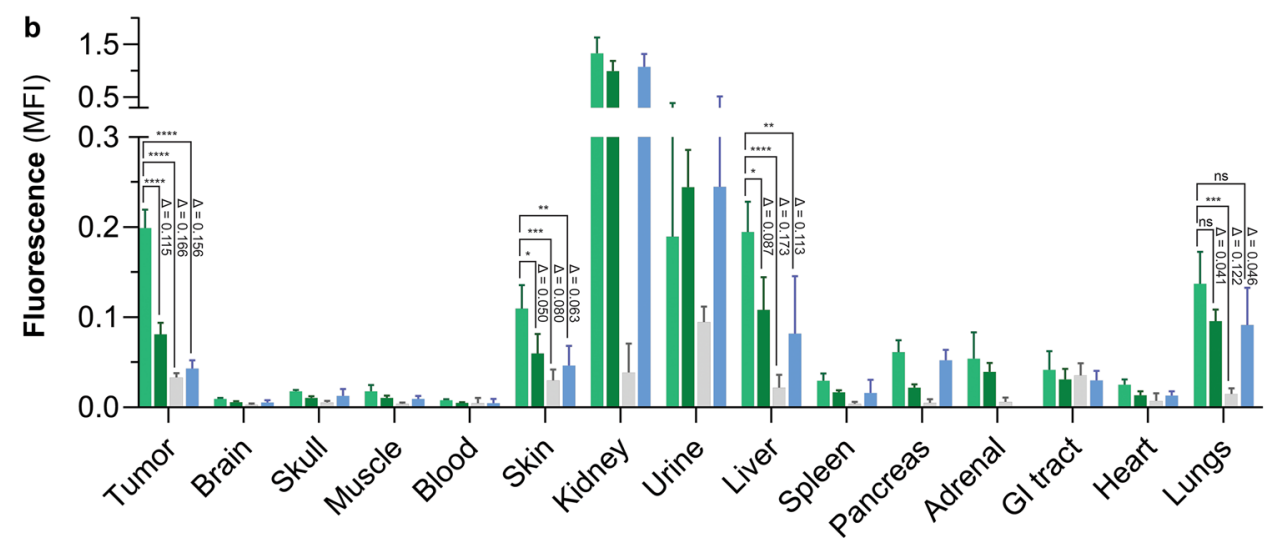

$$
\begin{array}{ll}
\text { H69 / 800CW-TATE } & \text { H69 / IRDye800CW } \\
\text { H69 / DOTA-TATE + 800CW-TATE } & \text { CH-157MN / 800CW-TATE }
\end{array}
$$

.

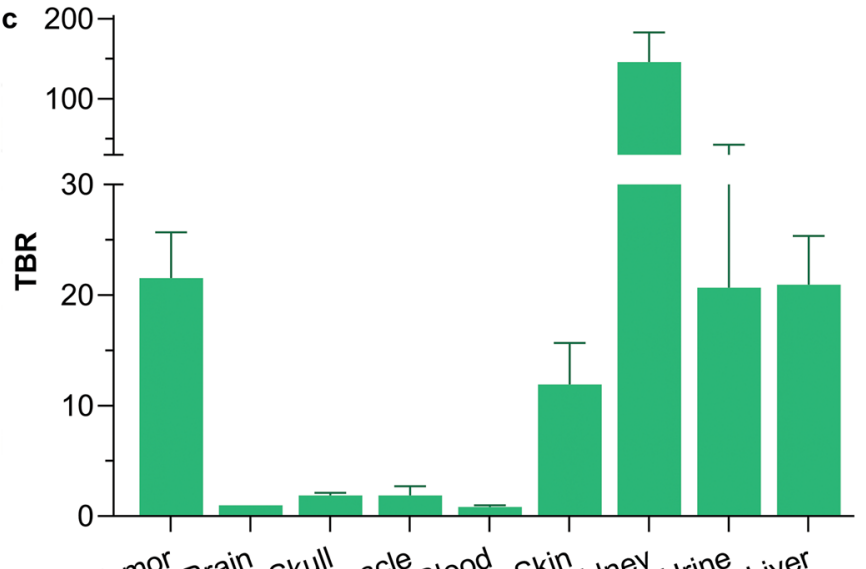

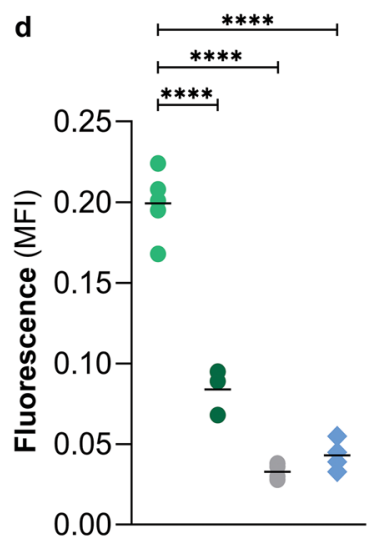

expression, which correlated with the low fluorescence intensity observed in these xenografts (Fig. 3c).

\section{Exploring MFGS on post-mortem mice}

To explore the use of 800CW-TATE in MFGS, we mimicked the procedure on a H69-xenografted mouse post-mortem. Tumor tissue could be distinguished during all steps of xenograft resection using fluorescence guidance. (Fig. 4) The TBR was 3.2. Using white light guidance, the tumor was almost completely resected. The remaining xenograft tissue could be observed using fluorescence guidance. Histological examination showed the resected tumor expressed $\mathrm{SSTR}_{2}$, whereas non-fluorescent non-tumorous background muscle tissue showed no $\mathrm{SSTR}_{2}$ expression, in line with absence of fluorescence. (Fig. 4b).

\section{$800 \mathrm{CW}$-TATE binding to human meningioma samples}

The binding properties of 800CW-TATE were determined on ten frozen meningioma patient samples. The meningioma 
a slide, white light

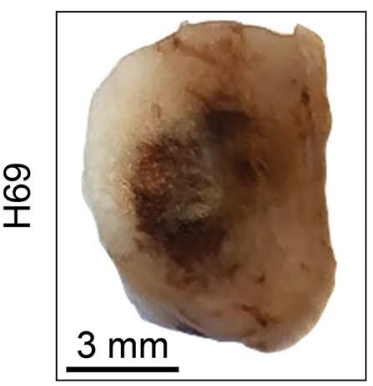

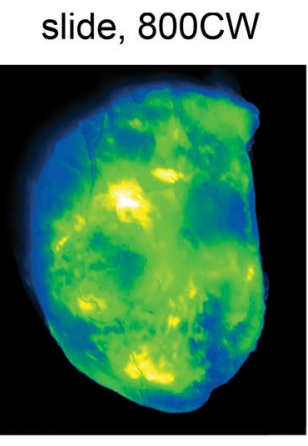

$4 \mu \mathrm{m}, 800 \mathrm{CW}$

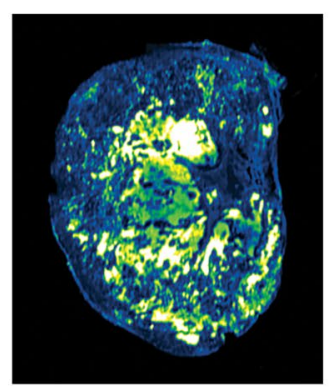

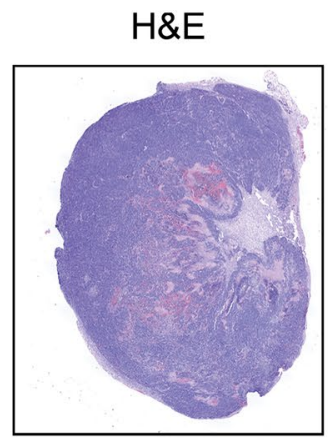

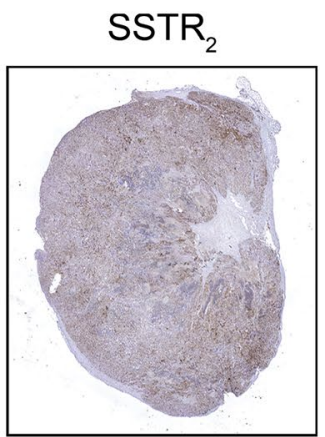

b

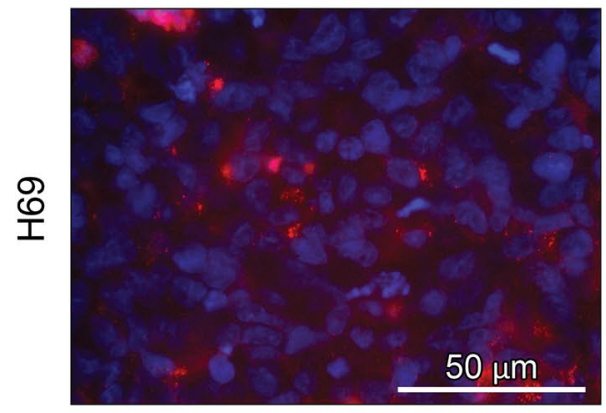

C

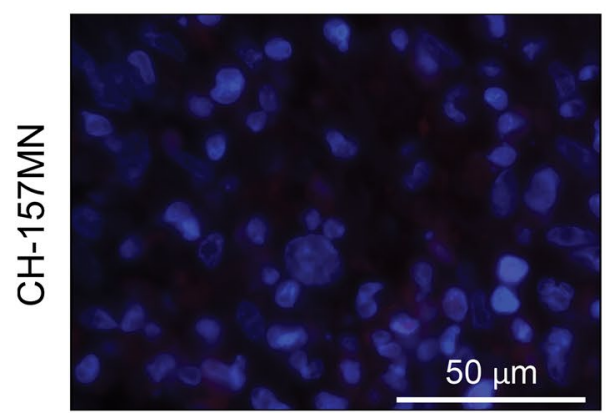

$H \& E$

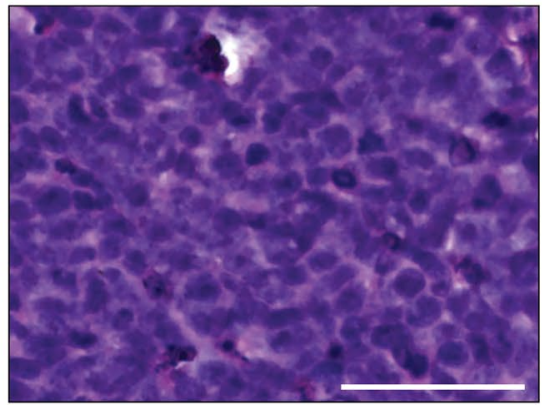

$H \& E$

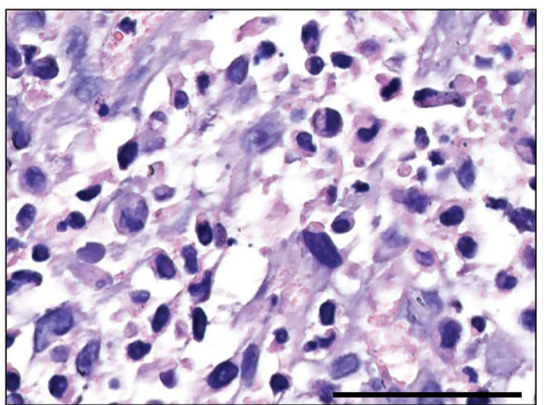

$\mathrm{SSTR}_{2}$

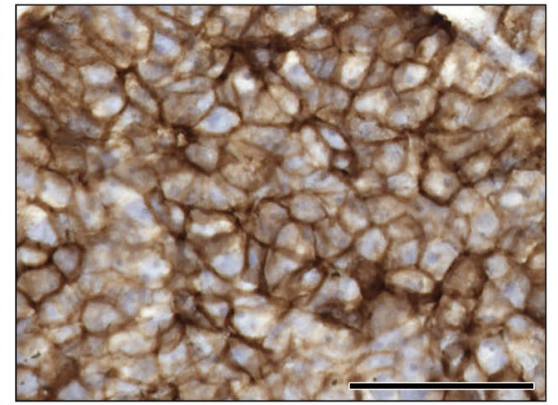

SSTR $_{2}$

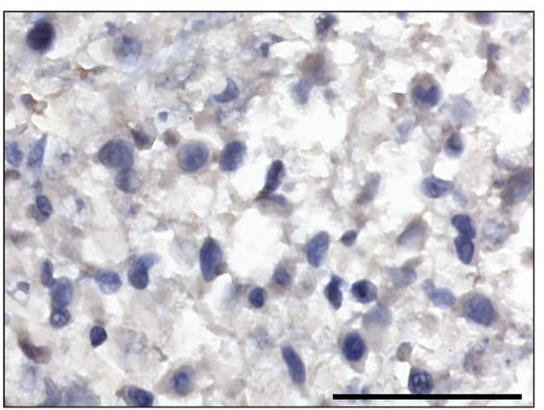

Fig. 3 800CW-TATE uptake and $\mathrm{SSTR}_{2}$ expression in xenografts. a Macroscopic overview of a $\mathrm{H} 69$ tumor slice as white light image, 800CW Odyssey CLx scan of the same slice and 800CW Odyssey CLx scan of a $4 \mu \mathrm{m}$ slide, $\mathrm{H} \& \mathrm{E}$, and $\mathrm{SSTR}_{2}$ counterstain. b 800CW-TATE fluorescence and $\mathrm{SSTR}_{2}$ expression are homogenous throughout the tumor. Micrographs of a H69 tumor slice incubated with $800 \mathrm{CW}-\mathrm{TATE}$ and stained with $\mathrm{H} \& \mathrm{E}$ and anti-SSTR 2 revealed a membranous and cytoplasmatic pattern for both 800CW-TATE and $\mathrm{SSTR}_{2}$. c CH-157MN xenograft showed low 800CW-TATE fluorescence and absent $\mathrm{SSTR}_{2}$ expression specimens, consisting of four WHO grade I, three WHO grade II and three WHO grade III samples, were evaluated for $\mathrm{SSTR}_{2}$ expression. Online Resource 5 shows patient characteristics and $\mathrm{SSTR}_{2}$ scores based on previous immunohistochemical data [7]. Incubation with 800CW-TATE showed fluorescence in all meningioma samples with membranous and cytoplasmic staining patterns. (Fig. 5a, left column) Consecutive sections were stained for $\mathrm{SSTR}_{2}$, revealing overall SSTR $_{2}$-positive staining at the same cellular localization as the $800 \mathrm{CW}-\mathrm{TATE}$ counterstain. (Fig. 5a, middle column) Fluorescence intensity of $800 \mathrm{CW}-\mathrm{TATE}$ was variable and did not correlate with WHO grades, but showed a positive trend between $\mathrm{SSTR}_{2}$ expression levels and fluorescence intensity.
Moreover, fresh meningioma samples (Online Resource 5) containing adjacent non-tumorous dura mater as well as meningioma tissue were analyzed for tracer binding and $\mathrm{SSTR}_{2}$ expression in order to evaluate selectivity for meningioma tissue. Preoperative imaging showed a convexity meningioma (Fig. 5b, left panel), which is depicted in the middle panel of Fig. 5b as a fresh specimen post resection. $\mathrm{SSTR}_{2}$ expression was strong in the meningioma bulk, but absent in adjacent dura mater which was non-tumorous confirmed by histopathology. (Fig. 5b, right panel; Online Resource 5) The same pattern was observed for $800 \mathrm{CW}$ TATE. (Fig. 5c) These results were comparable in the tested fresh samples. 
a
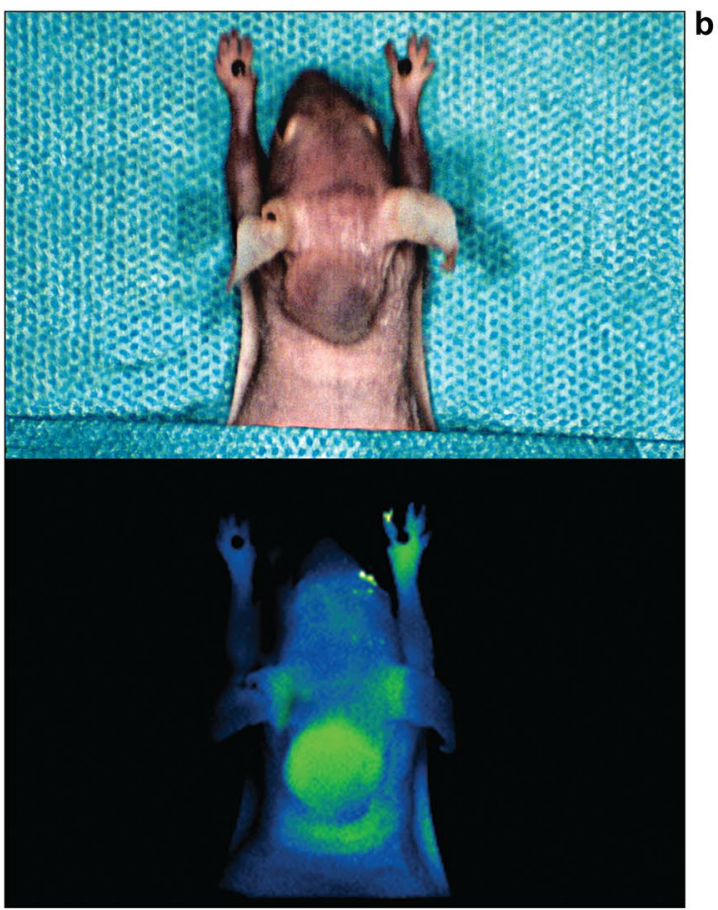

C

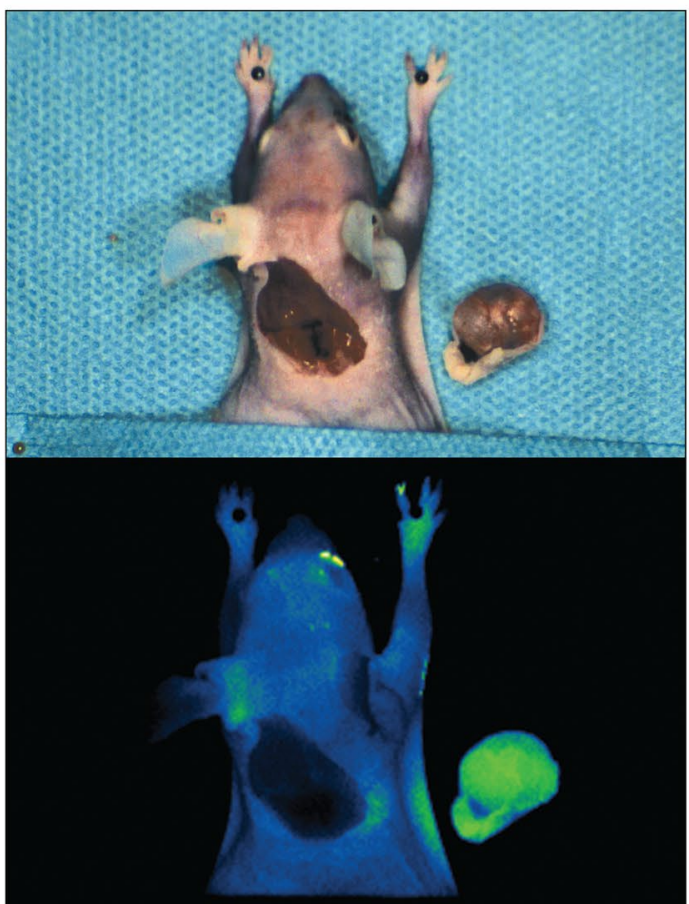

Fig. 4 Post-mortem MFGS on H69 xenografted mouse. Mouse bearing a H69 xenograft injected with 800CW-TATE underwent postmortem MFGS. The tumor was visualized prior (a) and during (b) xenograft removal. Small pieces of xenograft were initially unde-
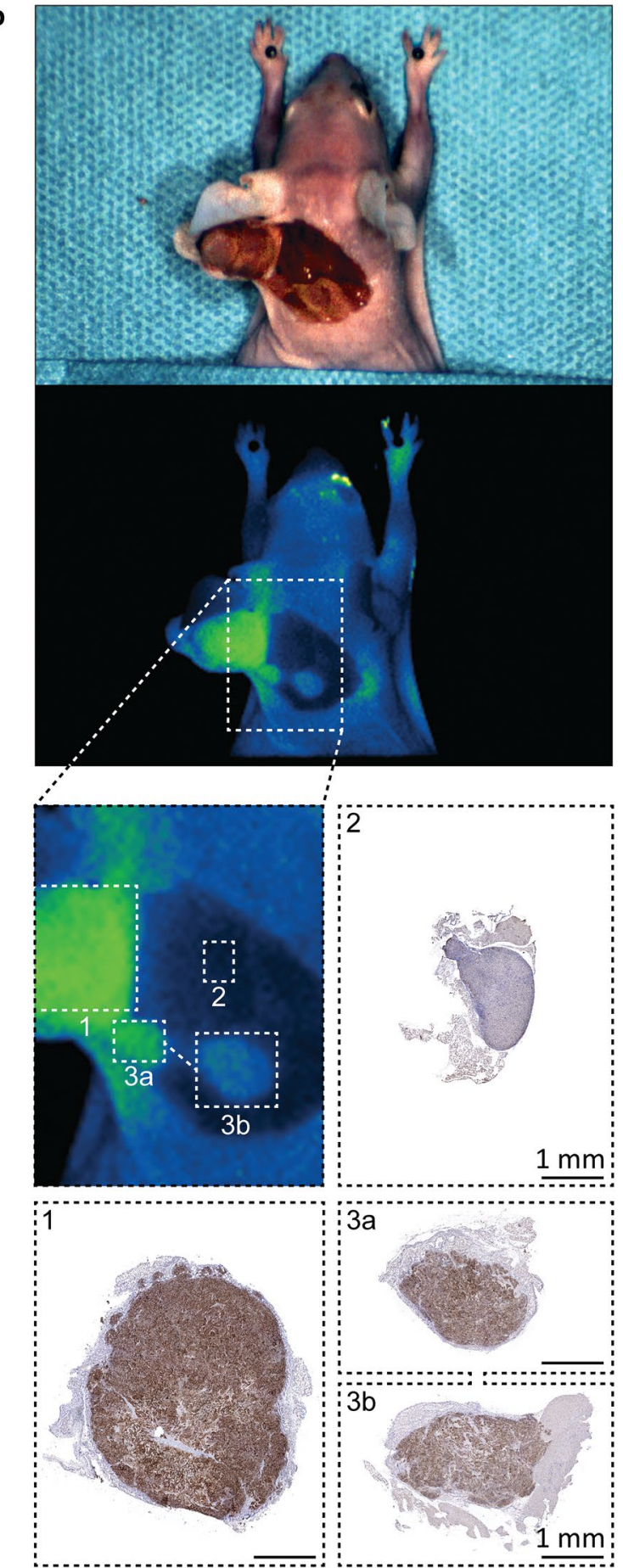

tected using white light guidance but could be readily observed using fluorescence. Fluorescent tissue showed $\mathrm{SSTR}_{2}$ expression, whereas non-fluorescent background tissue did not. Post resection, the wound bed shows low fluorescence intensity (c), with a TBR of 3.2 

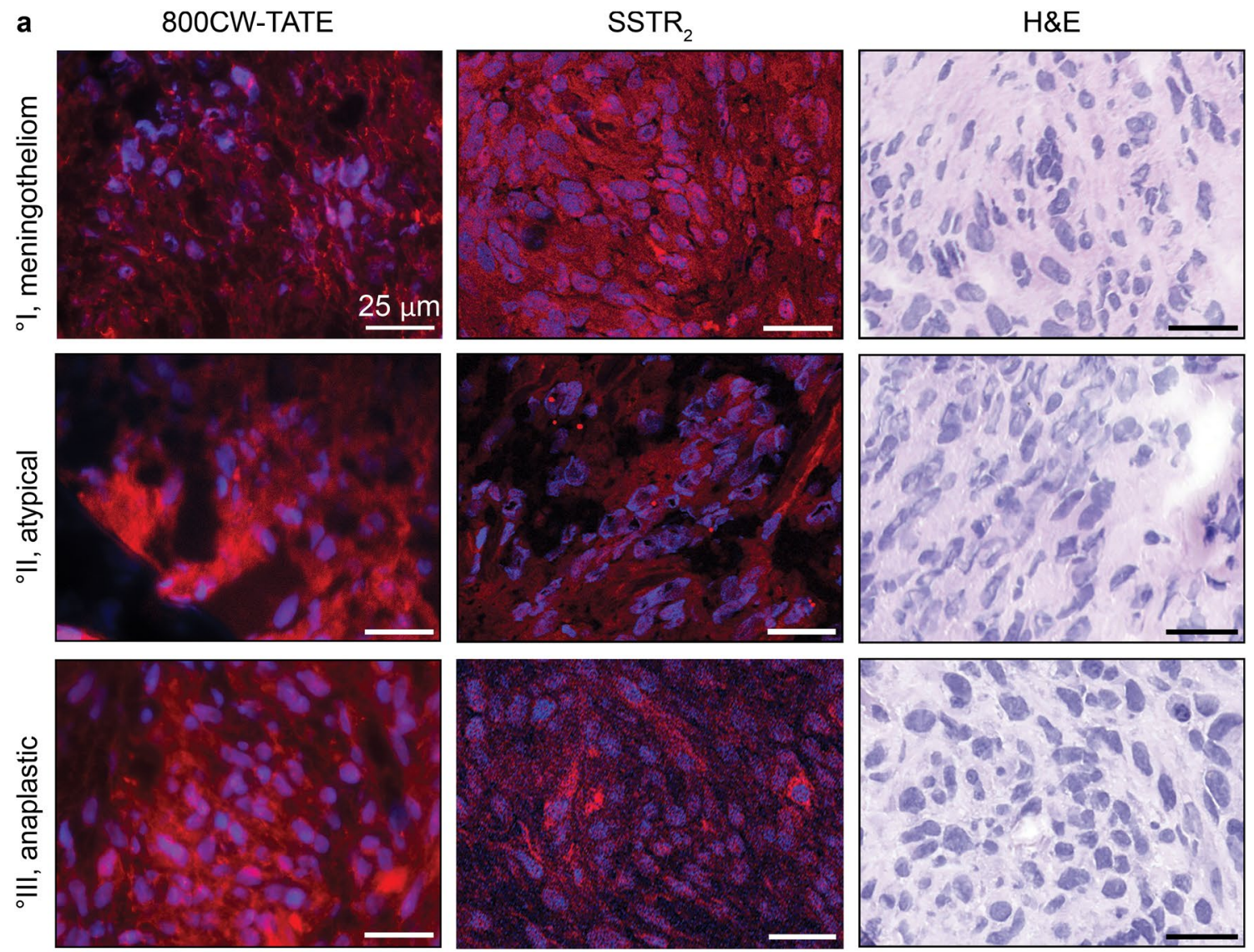

b

GE MRI

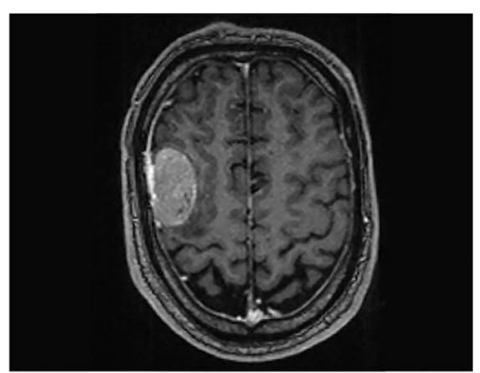

.

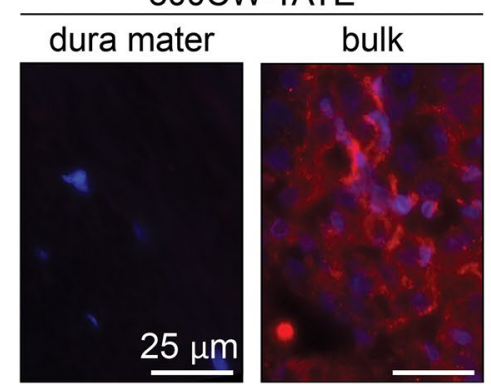

Whole specimen

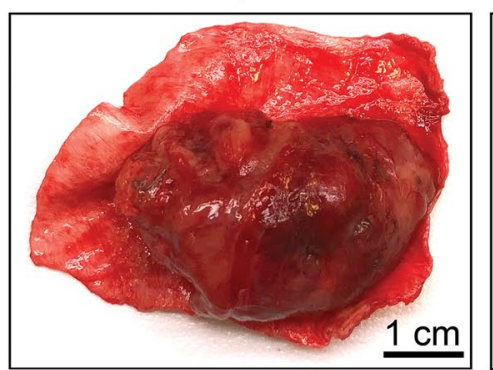

SSTR $_{2}$
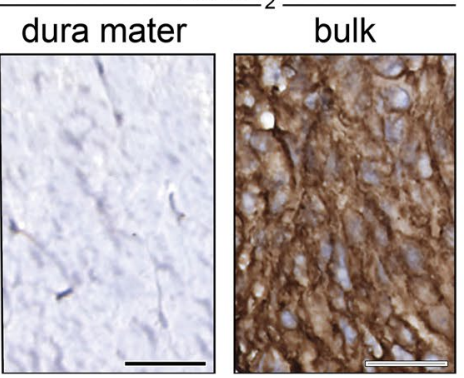

$\mathrm{SSTR}_{2}$

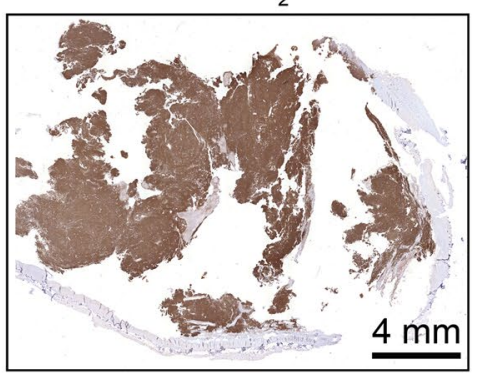

$H \& E$

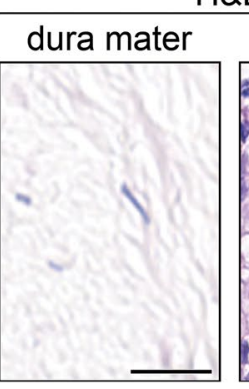

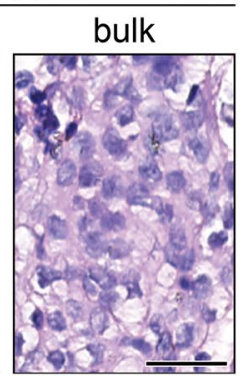


4Fig. 5 800CW-TATE uptake in clinical meningioma specimens. a Ten frozen meningioma specimens representing the different WHO grades were incubated with $800 \mathrm{CW}$-TATE. Micrographs of sequential sections stained for 800CW-TATE (left panel), SSTR 2 (middle panel), and H\&E (right panel). WHO I (top row), WHO II (middle row), and WHO III (bottom row) are depicted for each staining. All meningioma sections stained positive for $\mathrm{SSTR}_{2}$ and showed 800CW-TATE fluorescence regardless of WHO grade, with a membranous and cytoplasmatic staining pattern. A convexity meningioma revealed by GE MRI in a 71-year-old male (b, left panel) was resected (b, middle panel). After incubation with 800CW-TATE, the tumor bulk fluoresced, whereas the dura did not (c). SSTR 2 expression determined by IHC showed a strong membranous expression in the tumor bulk, but was absent in the dura mater, in concordance with tracer fluorescence (b, right panel; c)

\section{Discussion}

In this study we have generated a $\mathrm{SSTR}_{2}$-specific tracer, 800CW-TATE, and evaluated its potential for application in MFGS of meningiomas in xenograft mice models and clinical meningioma samples. The binding affinity of $800 \mathrm{CW}$ TATE was sufficient to proceed to tracer characterization. Displacement assays showed that DOTA-TATE has a higher affinity for $\mathrm{SSTR}_{2}$ than $800 \mathrm{CW}-\mathrm{TATE}$, however $\mathrm{IC}_{50}$ values for both were in the nanomolar range. The lower affinity for 800CW-TATE may be due to steric hindrance, an altered configuration and/or electrical charge of the tracer [23-25].

In this proof-of-principle study, xenografts of lung cancer $\mathrm{H} 69$ cells served as a $\mathrm{SSTR}_{2}$-positive tumor model [17-19]. The xenografts were heterotopically inoculated, creating a model that remained representative for meningiomas, because these intracranial tumors are located outside the blood-brain-barrier (BBB) and are all $\mathrm{SSTR}_{2}$ positive. Surprisingly, $\mathrm{CH}-157 \mathrm{MN}$ xenografts did not show $\mathrm{SSTR}_{2}$-expression, while $\mathrm{CH}-157 \mathrm{MN}$ cell cultures did express $\mathrm{SSTR}_{2}$ in line with our previous experience [7]. Thus, the H69 xenograft model provided a suitable model for SSTR $_{2}$ targeting, whereas the CH-157MN xenograft model eventually served as a SSTR ${ }_{2}$-negative control in this study. Clearly, the use of the $\mathrm{CH}-157 \mathrm{MN}$ xenograft model would have been ideal to further illustrate the suitability of 800CW-TATE for visualizing meningioma tissue, however, as an alternative strategy fresh frozen meningioma specimen were used, as discussed in more detail below.

In vivo, binding of $800 \mathrm{CW}$-TATE appeared to be $\mathrm{SSTR}_{2}$-mediated, as fluorescence uptake determined ex vivo on resected xenograft significantly decreased after a DOTATATE block and fluorescence was clearly lower in the $\mathrm{CH}$ $157 \mathrm{MN}$ xenografts. Although the block was not complete, this could be due to tracer binding to necrotic tissue, which we are currently investigating in a separate project. Comparison of tumor specific binding of 800CW-TATE to other IRDye $800 \mathrm{CW}$-coupled tracers is complicated due to the use of targeting vectors, imaging modalities and animal models described in literature. Previously, bevacizumabIRDye $800 \mathrm{CW}$ showed a $65 \%$ higher fluorescence signal compared with a non-targeted IgG-IRDye800CW control [26], which was in line with other studies applying various antibody-IRDye800CW labelled tracers [27-29]. Furthermore, several in vivo studies using octreotate-based tracers showed that tumor uptake of both $\left[{ }^{177} \mathrm{Lu}\right] \mathrm{Lu}$-DOTA-TATE and $\left[{ }^{213} \mathrm{Bi}\right] \mathrm{Bi}$-DOTA-TATE was blocked after the administration of DOTA-TATE $[17,18]$. Renal clearance of the tracer was observed by high fluorescence levels in kidneys and urine. In addition, tracer uptake was determined in liver, lungs, brain, muscle and blood. Uptake in these organs was mostly comparable to previously published reports applying $\left[{ }^{111} \mathrm{In}\right] \mathrm{In}$-DOTA-TATE, $\left[{ }^{213} \mathrm{Bi}\right] \mathrm{Bi}$-DOTA-TATE, $\left[{ }^{111} \mathrm{In}\right]$ In-DTPA-TATE or Cy5-[ $\left.{ }^{111} \mathrm{In}\right]$ In-DTPA-TATE [17-19]. Lastly, IRDye800CW-carboxylate-treated H69 xenograft bearing mice had very low uptake of the fluorescent dye, indicating that tumor fluorescence is not related to freely circulating dye in our study. This is in agreement with other reports showing no tumor-specific uptake of IRDye800CW in a colon cancer model and orthotopic glioma model [30, 31].

The ex vivo TBR of 21.1 in our present experiments is encouraging for future clinical translation, based on our experience in both preclinical and clinical intra-operative imaging settings [32, 33]. Fluorescence was low in neurosurgical background tissues such as brain, skull and muscle, allowing for clear distinction and visualization of tumor tissue. This is highly relevant when using 800CW-TATE for resection of intracranial meningioma. Tracer fluorescence detected in the skin is usually not of importance during neurosurgery because the skin flap is retracted out of the operative area and draped. However, the relevance of background fluorescence from the skin in patients with meningiomainvasion into the skull and (sub) cutaneous tissues should be evaluated in future clinical MFGS studies. Our post-mortem MFGS experiment on a H69 xenograft bearing mouse achieved a TBR of 3.2 and small xenograft residues could be easily detected. In our experience, various clinical imaging systems for fluorescence guided surgery require a TBR $>2.0$ to be able to be of value in a clinical setting.

Lastly, the clinical meningioma specimens (representing different WHO grades) all expressed SSTR $_{2}$ and showed 800CW-TATE-specific fluorescence staining. Importantly, 800CW-TATE could distinguish meningioma from dura mater on freshly resected specimens. The observed variation in fluorescence intensity of both stainings varied among WHO grades, which is not in line with larger sample sizes available in literature and our previous results where we found no significant differences in $\mathrm{SSTR}_{2}$-expression among WHO grades [7-11]. Nevertheless, the current results illustrate that $800 \mathrm{CW}-\mathrm{TATE}$ binds to all meningioma grades. 
Future in-human trials will further elucidate the relationship between $\mathrm{SSTR}_{2}$ expression and 800CW-TATE fluorescence.

$\mathrm{SSTR}_{2}$-targeted tracers have already been applied for diagnostics and treatment of meningiomas. For example, in tumor imaging ${ }^{111}$ In-octreotide SPECT in combination with MRI has been successfully used to differentiate meningioma from other intracranial lesions [34]. ${ }^{68}$ Ga-DOTATOC- and ${ }^{68}$ Ga-DOTA-TATE-PET/MRI provided better imaging quality during follow-up of intracranial meningiomas and improved the ability of detection of residual meningioma or tumor recurrence after initial treatment [35, 36]. Ongoing trials further investigate diagnostic and followup imaging feasibility of SSTR ${ }_{2}$-targeted radiopeptides in meningioma (NCT04298541, NCT03953131). Furthermore, peptide receptor radiotherapy with ${ }^{90} \mathrm{Y}$ - and ${ }^{177} \mathrm{Lu}$-DOTATOC has resulted in patients with inoperable meningioma reaching stable disease [37]. Currently, ongoing phase II trials with among others ${ }^{177}$ Lu-DOTA-TATE (Lutathera, NCT04082520), ${ }^{90}$ Y-DOTA-TOC and ${ }^{90}$ Y-DOTA-TATE (NCT03273712) will further demonstrate the value of this treatment modality for inoperable, progressive meningiomas. Our next step in the development of 800CW-TATE for MFGS in meningiomas will be the clinical evaluation of this tracer in patients with intracranial meningiomas, aiming to add another $\mathrm{SSTR}_{2}$-directed approach to the treatment of this type of tumors.

Dural or bone thickening may be indicative of meningioma invasion, but can also be caused by vascular congestion or reactive tissue changes. Furthermore, meningioma invasion may be present in these adjacent tissues without causing macroscopically detectible differences using white light. This can lead to a (yet undetected) subtotal resection, which is a major risk factor for recurrence [2]. Currently applied dyes, such as 5-ALA, ICG and fluorescein, have a too low sensitivity and/or specificity to distinguish meningioma invasion from reactive changes or even from normal tissue of the adjacent dura and bone. 800CW-TATE targets $\mathrm{SSTR}_{2}$ which is not expressed in normal bone or dura mater. Thus, in a future clinical study we expect that with the aid of this tracer and technique, not only small tumor remnants or satellite lesions will be detected during primary or recurrent tumor resection, but meningioma invasion status of adjacent soft and bone tissue will be determined more accurately as well.

\section{Conclusions}

800CW-TATE appears to be a promising fluorescent dye for MFGS of intracranial meningiomas. It has the potency to aid in the safer and completer resection, which is especially relevant in the high grade meningiomas or in meningiomas located in difficult anatomical locations.

\section{Future perspectives}

With these encouraging preclinical results, a dose-finding clinical trial is feasible. Indeed, both octreotate and IRDye $800 \mathrm{CW}$ have been applied numerous times in humans, increasing the chances of successful clinical translation [16, $38,39]$. For a first in-human trial, a microdosing study with $800 \mathrm{CW}-T A T E$ in a small number of patients would be the next step. In line with our preclinical study results regarding timing of tracer administration, tumor uptake and imaging, intravenous tracer administration in patients could take place approximately four hours prior to surgical incision, but can be adjusted to obtain optimal TBRs. Altogether, $800 \mathrm{CW}$ TATE shows a favorable biodistribution for application in a neurosurgical setting, with the ultimate goal of improving curative resection rates in patients with intracranial meningioma.

Supplementary Information The online version contains supplementary material available at https://doi.org/10.1007/s11060-021-03739-1.

Acknowledgements The authors would like to thank several contributors in the University Medical Center Groningen: dr Frans V. Suurs for his useful discussions regarding study design and data acquisition; the microsurgical team and animal caretakers of the animal facility for biotechnical assistance; dr ir Kees W.A. van der Kolk for useful comments regarding in vivo imaging; Pieter J. Steinkamp, MD for his help with the SurgVision; Matthijs D. Linssen, PharmD for pharmacological advise; the pathology assistants for advice and guidance on fixating and embedding; mr Gert Jan Meersma and mrs Tineke van der Sluis for assisting with ex vivo imaging and in vitro studies; and Marina López-Álvarez, MSc for assistance with fluorescence microscopy. In the Erasmus MC, Rotterdam, dr Yann Seimbille and dr Erik de Blois contributed to discussions regarding radiolabeling; Eline Ruigrok, MSc supported performing autoradiography studies; mr Yanto Ridwan instructed and advised concerning the use of the FMT2500.

Author contributions All authors contributed to the study conception and design. Material preparation and data collection were performed by BD, MS, FA, SD, and ME. Data analysis was performed by BD, MJ, SK, JN, WD, FK, and RG. The first draft of the manuscript was written by BD and all authors commented on previous versions of the manuscript. All authors read and approved the final manuscript and agree with its submission to the Journal of Neuro-Oncology.

Funding BMD is supported by research grants of the University of Groningen, the University Medical Center Groningen, and the de CockHadders Stichting. ME was financially supported by ERC Consolidator Grant \#682421-Tension.

Data availability The datasets generated during and/or analyzed during the current study are available from the corresponding author on reasonable request.

\section{Declarations}

Conflict of interest WdD was member of the advisory board for GBM diagnostic testing for AbbVie. MdJ and JN have an unrelated investigator-initiated project contract with Advanced Accelerator Applications, 
a Novartis company. FA is CEO of piCHEM Forschungs und EntwicklungsGmbH, Raaba-Grambach, Graz, Austria.

Consent to participate Informed consent was obtained from all individual participants included in the study. If informed consent could not be obtained, the Code of Conduct for responsible use of human tissue was followed.

Consent for publication Patients signed informed consent regarding publishing their anonymized data and photographs.

Ethical approval Ethics of animal and human research subjects has been reviewed by the Institutional Review Board of the University of Groningen and University Medical Centre Groningen. Animal care complied with the Guide for the Care and Use of Laboratory Animals. For patient material, the Board approved the experimental protocol and informed consent.

Open Access This article is licensed under a Creative Commons Attribution 4.0 International License, which permits use, sharing, adaptation, distribution and reproduction in any medium or format, as long as you give appropriate credit to the original author(s) and the source, provide a link to the Creative Commons licence, and indicate if changes were made. The images or other third party material in this article are included in the article's Creative Commons licence, unless indicated otherwise in a credit line to the material. If material is not included in the article's Creative Commons licence and your intended use is not permitted by statutory regulation or exceeds the permitted use, you will need to obtain permission directly from the copyright holder. To view a copy of this licence, visit http://creativecommons.org/licenses/by/4.0/.

\section{References}

1. Louis DN, Perry A, Reifenberger G et al (2016) The 2016 world health organization classification of tumors of the central nervous system: a summary. Acta Neuropathol 131:803-820. https://doi. org/10.1007/s00401-016-1545-1

2. Campbell BA, Jhamb A, Maguire JA et al (2009) Meningiomas in 2009. Controversies and future challenges. Am J Clin Oncol Cancer Clin Trials 32:73-85. https://doi.org/10.1097/COC.0b013 e31816fc920

3. McMullen KP, Stieber VW (2004) Meningioma: current treatment options and future directions. Curr Treat Options Oncol 5:499-509. https://doi.org/10.1007/s11864-004-0038-y

4. Simpson D (1957) The recurrence of intracranial meningiomas after surgical treatment. J Neurol Neurosurg Psychiat 20:22-39

5. Whittle IR, Smith C, Navoo P, Collie D (2004) Meningiomas. Lancet 363:1535-1543. https://doi.org/10.1016/S0140-6736(04) 16153-9

6. Esser JP, Krenning EP, Teunissen JJM et al (2006) Comparison of [(177)Lu-DOTA(0), Tyr(3)]octreotate and [(177)Lu-DOTA(0), $\operatorname{Tyr}(3)$ ]octreotide: which peptide is preferable for PRRT? Eur J Nucl Med Mol Imaging 33:1346-1351. https://doi.org/10.1007/ s00259-006-0172-9

7. Dijkstra BM, Motekallemi A, Den Dunnen WFA et al (2018) SSTR-2 as a potential tumour-specific marker for fluorescenceguided meningioma surgery. Acta Neurochir 160:1539-1546. https://doi.org/10.1007/s00701-018-3575-z

8. Boulagnon-Rombi C, Fleury C, Fichel C et al (2017) Immunohistochemical approach to the differential diagnosis of meningiomas and their mimics. J Neuropathol Exp Neurol 76:289-298. https:// doi.org/10.1093/jnen/nlx008
9. de Oliveira Silva CB, Ongaratti BR, Trott G et al (2015) Expression of somatostatin receptors (SSTR1-SSTR5) in meningiomas and its clinicopathological significance. Int J Clin Exp Pathol 8:13185-13192

10. Menke JR, Raleigh DR, Gown AM et al (2015) Somatostatin receptor $2 \mathrm{a}$ is a more sensitive diagnostic marker of meningioma than epithelial membrane antigen. Acta Neuropathol 130:441443. https://doi.org/10.1007/s00401-015-1459-3

11. Norden AD, Drappatz J, Wen PY (2007) Targeted drug therapy for meningiomas. Neurosurg Focus 23:E12. https://doi.org/10.3171/ FOC-07/10/E12

12. Reubi JC, Schar JC, Waser B et al (2000) Affinity profiles for human somatostatin receptor subtypes SST1-SST5 of somatostatin radiotracers selected for scintigraphic and radiotherapeutic use. Eur J Nucl Med 27:273-282

13. Harlaar NJ, Koller M, de Jongh SJ et al (2016) Molecular fluorescence-guided surgery of peritoneal carcinomatosis of colorectal origin: a single-centre feasibility study. Lancet Gastroenterol Hepatol 1:283-290. https://doi.org/10.1016/S2468-1253(16) 30082-6

14. Nagengast WB, Hartmans E, Garcia-Allende PB et al (2019) Nearinfrared fluorescence molecular endoscopy detects dysplastic oesophageal lesions using topical and systemic tracer of vascular endothelial growth factor A. Gut 68:7-10. https://doi.org/10.1136/ gutjnl-2017-314953

15. Ter Weele EJ, Terwisscha van Scheltinga AGT, Linssen MD et al (2016) Development, preclinical safety, formulation, and stability of clinical grade bevacizumab-800CW, a new near infrared fluorescent imaging agent for first in human use. Eur J Pharm Biopharm 104:226-234. https://doi.org/10.1016/j.ejpb.2016.05. 008

16. Strosberg J, El-Haddad G, Wolin E et al (2017) Phase 3 Trial of ${ }^{177} \mathrm{Lu}$-Dotatate for Midgut neuroendocrine tumors. N Engl J Med 376:125-135. https://doi.org/10.1056/NEJMoa1607427

17. Dalm SU, Haeck J, Doeswijk GN et al (2017) SSTR-mediated imaging in breast cancer: is there a role for radiolabeled somatostatin receptor antagonists? J Nucl Med 58:1609-1614. https:// doi.org/10.2967/jnumed.116.189035

18. Chan HS, Konijnenberg MW, de Blois E et al (2016) Influence of tumour size on the efficacy of targeted alpha therapy with (213) Bi-[DOTA(0), Tyr(3)]-octreotate. EJNMMI Res 6:6. https://doi. org/10.1186/s13550-016-0162-2

19. Santini C, Kuil J, Bunschoten A et al (2016) Evaluation of a fluorescent and radiolabeled hybrid somatostatin analog in vitro and in mice bearing H69 neuroendocrine xenografts. J Nucl Med 57:1289-1295. https://doi.org/10.2967/jnumed.115.164970

20. Tsai JC, Goldman CK, Gillespie GY (1995) Vascular endothelial growth factor in human glioma cell lines: induced secretion by EGF, PDGF-BB, and bFGF. J Neurosurg 82:864-873. https://doi. org/10.3171/jns.1995.82.5.0864

21. de Jong M, Breeman WAP, Bakker WH et al (1998) Comparison of (111)In-labeled somatostatin analogues for tumor scintigraphy and radionuclide therapy. Cancer Res 58:437-441

22. Chan HS, de Blois E, Morgenstern A et al (2017) In Vitro comparison of $213 \mathrm{Bi}$ - and $177 \mathrm{Lu}$-radiation for peptide receptor radionuclide therapy. PLoS ONE 12:e0181473. https://doi.org/10.1371/ journal.pone.0181473

23. Froidevaux S, Eberle AN, Christe $M$ et al (2002) Neuroendocrine tumor targeting: study of novel gallium-labeled somatostatin radiopeptides in a rat pancreatic tumor model. Int J cancer 98:930-937

24. Yim C-B, van der Wildt B, Dijkgraaf I et al (2011) Spacer effects on in vivo properties of DOTA-conjugated dimeric [Tyr3] octreotate peptides synthesized by a "Cu(I)-click" and "sulfo-click" ligation method. ChemBioChem 12:750-760. https://doi.org/10.1002/ cbic. 201000639 
25. Parry JJ, Kelly TS, Andrews R, Rogers BE (2007) In vitro and in vivo evaluation of 64Cu-labeled DOTA-linker-bombesin(7-14) analogues containing different amino acid linker moieties. Bioconjug Chem 18:1110-1117. https://doi.org/10.1021/bc0603788

26. Terwisscha van Scheltinga AGT, van Dam GM, Nagengast WB et al (2011) Intraoperative near-infrared fluorescence tumor imaging with vascular endothelial growth factor and human epidermal growth factor receptor 2 targeting antibodies. J Nucl Med 52:1778-1785. https://doi.org/10.2967/jnumed.111.092833

27. Heath CH, Deep NL, Sweeny L et al (2012) Use of panitumumabIRDye800 to image microscopic head and neck cancer in an orthotopic surgical model. Ann Surg Oncol 19:3879-3887. https://doi. org/10.1245/s10434-012-2435-y

28. Day KE, Sweeny L, Kulbersh B et al (2013) Preclinical comparison of near-infrared-labeled cetuximab and panitumumab for optical imaging of head and neck squamous cell carcinoma. Mol imaging Biol 15:722-729. https://doi.org/10.1007/ s11307-013-0652-9

29. van Driel PBAA, Boonstra MC, Prevoo HAJM et al (2016) EpCAM as multi-tumour target for near-infrared fluorescence guided surgery. BMC Cancer 16:884. https://doi.org/10.1186/ s12885-016-2932-7

30. Hollandsworth HM, Lwin TM, Amirfakhri S et al (2019) Anticlaudin-1 conjugated to a near-infrared fluorophore targets colon cancer in PDOX mouse models. J Surg Res 242:145-150. https:// doi.org/10.1016/j.jss.2019.04.048

31. Zhou H, Luby-Phelps K, Mickey BE et al (2009) Dynamic nearinfrared optical imaging of 2-deoxyglucose uptake by intracranial glioma of athymic mice. PLoS ONE 4:e8051. https://doi.org/10. 1371/journal.pone.0008051

32. Koller M, Qiu S-Q, Linssen MD et al (2018) Implementation and benchmarking of a novel analytical framework to clinically evaluate tumor-specific fluorescent tracers. Nat Commun 9:3739. https://doi.org/10.1038/s41467-018-05727-y
33. Steinkamp PJ, Pranger BK, Li M et al (2020) Fluorescenceguided visualization of soft tissue sarcomas by targeting vascular endothelial growth factor-A: a phase 1 single-center clinical trial. J Nucl Med. https://doi.org/10.2967/jnumed.120.245696

34. Nathoo N, Ugokwe K, Chang AS et al (2007) The role of 111indium-octreotide brain scintigraphy in the diagnosis of cranial, dural-based meningiomas. J Neurooncol 81:167-174. https:// doi.org/10.1007/s11060-006-9210-5

35. Ivanidze J, Roytman M, Lin E et al (2019) Gallium-68 DOTATATE PET in the evaluation of intracranial meningiomas. J Neuroimaging 29:650-656. https://doi.org/10.1111/jon.12632

36. Afshar-Oromieh A, Wolf MB, Kratochwil C et al (2015) Comparison of $68 \mathrm{Ga}$-DOTATOC-PET/CT and PET/MRI hybrid systems in patients with cranial meningioma: initial results. Neuro Oncol 17:312-319. https://doi.org/10.1093/neuonc/nou131

37. Marincek N, Radojewski P, Dumont RA et al (2015) Somatostatin receptor-targeted radiopeptide therapy with 90Y-DOTATOC and 177Lu-DOTATOC in progressive meningioma: long-term results of a phase II clinical trial. J Nucl Med 56:171-176. https://doi.org/ 10.2967/jnumed.114.147256

38. Zhang RR, Schroeder AB, Grudzinski JJ et al (2017) Beyond the margins: real-time detection of cancer using targeted fluorophores. Nat Rev Clin Oncol 14:347-364. https://doi.org/10.1038/nrcli nonc. 2016.212

39. Marshall MV, Draney D, Sevick-Muraca EM, Olive DM (2010) Single-dose intravenous toxicity study of IRDye $800 \mathrm{CW}$ in sprague-dawley Rats. Mol Imaging Biol 12:583-594. https://doi. org/10.1007/s11307-010-0317-x

Publisher's Note Springer Nature remains neutral with regard to jurisdictional claims in published maps and institutional affiliations.

\title{
Authors and Affiliations
}

\author{
Bianca M. Dijkstra ${ }^{1}(1) \cdot$ Marion de Jong ${ }^{2} \cdot$ Marcus C. M. Stroet ${ }^{2,3}$. Fritz Andreae ${ }^{4}$. Sebastiaan E. Dulfer ${ }^{1}$. \\ Marieke Everts $^{5}$. Schelto Kruijff ${ }^{6}$. Julie Nonnekens $s^{2,3}$. Wilfred F. A. den Dunnen ${ }^{7}$. Frank A. E. Kruyt ${ }^{5}$ (i) \\ Rob J. M. Groen ${ }^{1}$ (I)
}

1 Department of Neurosurgery, University of Groningen, University Medical Center Groningen, Hanzeplein 1, P.O. Box 30.001, 9700 VB Groningen, The Netherlands

2 Department of Radiology and Nuclear Medicine, Erasmus MC, Rotterdam, The Netherlands

3 Department of Molecular Genetics, Oncode Institute, Erasmus MC, Rotterdam, The Netherlands

4 piCHEM Forschungs und EntwicklungsGmbH, Raaba-Grambach, Graz, Austria
5 Department of Medical Oncology, University of Groningen, University Medical Center Groningen, Groningen, The Netherlands

6 Department of Surgery, University of Groningen, University Medical Center Groningen, Groningen, The Netherlands

7 Department of Pathology and Medical Biology, University of Groningen, University Medical Center Groningen, Groningen, The Netherlands 